\title{
A NOTE ON BIHARMONIC CURVES IN SASAKIAN SPACE FORMS
}

\author{
DOREL FETCU
}

\begin{abstract}
We classify the biharmonic non-Legendre curves in a Sasakian space form for which the angle between the tangent vector field and the characteristic vector field is constant and obtain explicit examples of such curves in $\mathbb{R}^{2 n+1}(-3)$.
\end{abstract}

\section{INTRODUCTION}

In 1964, J. Eells and J.H. Sampson introduced the notion of poly-harmonic maps as a natural generalization of harmonic maps ([8]). Thus, while harmonic maps between Riemannian manifolds $\phi:(M, g) \rightarrow(N, h)$ are the critical points of the energy functional $E(\phi)=\frac{1}{2} \int_{M}|d \phi|^{2} v_{g}$, the biharmonic maps are the critical points of the bienergy functional $E_{2}(\phi)=\frac{1}{2} \int_{M}|\tau(\phi)|^{2} v_{g}$.

On the other hand, B.-Y. Chen defined the biharmonic submanifolds in an Euclidean space as those with harmonic mean curvature vector field ([6]). If we apply the characterization formula of biharmonic maps to Riemannian immersions into Euclidean spaces, we recover Chen's notion of biharmonic submanifold.

The Euler-Lagrange equation for the energy functional is $\tau(\phi)=0$, where $\tau(\phi)=$ trace $\nabla d \phi$ is the tension field, and the Euler-Lagrange equation for the bienergy functional was derived by G. Y. Jiang in [13]:

$$
\begin{aligned}
\tau_{2}(\phi) & =-\Delta \tau(\phi)-\operatorname{trace} R^{N}(d \phi, \tau(\phi)) d \phi \\
& =0 .
\end{aligned}
$$

Since any harmonic map is biharmonic, we are interested in non-harmonic biharmonic maps, which are called proper-biharmonic.

There are several classification results and some methods to construct biharmonic submanifolds in space forms ([14], [2]). In a natural way, the next step is the study of biharmonic submanifolds in Sasakian space forms. Thus, J. Inoguchi classified in 12 the proper-biharmonic Legendre curves and Hopf cylinders in a 3-dimensional Sasakian space form $M^{3}(c)$, and in [9] the explicit parametric equations were obtained. In [7], J.T. Cho, J. Inoguchi and J.-E. Lee studied the biharmonic curves in a 3-dimensional Sasakian space forms and T. Sasahara studied the biharmonic integral surfaces in 5-dimensional Sasakian space forms ([18]). New classification results for biharmonic Legendre curves and examples of proper-biharmonic submanifolds in any dimensional Sasakian space form were obtained in [10].

Recent results on biharmonic submanifolds in spaces of nonconstant sectional curvature were obtained by T. Ichiyama, J. Inoguchi and H. Urakawa in [11, by Y.-L. Ou and Z.-P. Wang in [15], and by W. Zhang in [20].

2000 Mathematics Subject Classification. 53C42, 53B25.

Key words and phrases. Biharmonic curves, Sasakian space forms.

The author wants to thank to Prof. C. Oniciuc for many discussions and useful suggestions.

The author was partially supported by the Grant CEEX, ET, 5871/2006, Romania. 
Biharmonic submanifolds in pseudo-Euclidean spaces were also studied, and many examples and classification results were obtained (for example, see [1], 6]).

The goals of our paper are to obtain new classification results for biharmonic non-Legendre curves in any dimensional Sasakian space form and to obtain explicit equations for some of such curves in $\mathbb{R}^{2 n+1}(-3)$.

For a general account of biharmonic maps see [14] and The Bibliography of Biharmonic Maps [19].

Conventions. We work in the $C^{\infty}$ category, that means manifolds, metrics, connections and maps are smooth. The Lie algebra of the vector fields on $M$ is denoted by $C(T M)$.

\section{Preliminaries}

A triple $(\varphi, \xi, \eta)$ is called a contact structure on a manifold $N^{2 n+1}$, where $\varphi$ is a tensor field of type $(1,1)$ on $N, \xi$ is a vector field and $\eta$ is an 1-form, if

$$
\varphi^{2}=-I+\eta \otimes \xi, \quad \eta(\xi)=1, \quad \forall X, Y \in C(T N) .
$$

A Riemannian metric $g$ on $N$ is said to be an associated metric and then $(N, \varphi, \xi, \eta, g)$ is a contact metric manifold if

$$
g(\varphi X, \varphi Y)=g(X, Y)-\eta(X) \eta(Y), \quad g(X, \varphi Y)=d \eta(X, Y), \quad \forall X, Y \in C(T N) .
$$

A contact metric structure $(\varphi, \xi, \eta, g)$ is called normal if

$$
N_{\varphi}+2 d \eta \otimes \xi=0
$$

where

$$
N_{\varphi}(X, Y)=[\varphi X, \varphi Y]-\varphi[\varphi X, Y]-\varphi[X, \varphi Y]+\varphi^{2}[X, Y], \quad \forall X, Y \in C(T N),
$$

is the Nijenhuis tensor field of $\varphi$.

A contact metric manifold $(N, \varphi, \xi, \eta, g)$ is a Sasakian manifold if it is normal or, equivalently, if

$$
\left(\nabla_{X} \varphi\right)(Y)=g(X, Y) \xi-\eta(Y) X, \quad \forall X, Y \in C(T N)
$$

The contact distribution of a Sasakian manifold $(N, \varphi, \xi, \eta, g)$ is defined by $\{X \in$ $T N: \eta(X)=0\}$, and an integral curve of the contact distribution is called Legendre curve.

Let $(N, \varphi, \xi, \eta, g)$ be a Sasakian manifold. The sectional curvature of a 2-plane generated by $X$ and $\varphi X$, where $X$ is an unit vector orthogonal to $\xi$, is called $\varphi$ sectional curvature determined by $X$. A Sasakian manifold with constant $\varphi$-sectional curvature $c$ is called a Sasakian space form and it is denoted by $N(c)$. The curvature tensor field of a Sasakian space form $N(c)$ is given by

$$
\begin{aligned}
R(X, Y) Z= & \frac{c+3}{4}\{g(Z, Y) X-g(Z, X) Y\}+\frac{c-1}{4}\{\eta(Z) \eta(X) Y- \\
& -\eta(Z) \eta(Y) X+g(Z, X) \eta(Y) \xi-g(Z, Y) \eta(X) \xi+ \\
& +g(Z, \varphi Y) \varphi X-g(Z, \varphi X) \varphi Y+2 g(X, \varphi Y) \varphi Z\} .
\end{aligned}
$$

\section{Biharmonic NOn-Legendre CURVES in SASAKIAN SPACE FORMS}

Definition 3.1. Let $\left(N^{m}, g\right)$ be a Riemannian manifold and $\gamma: I \rightarrow N$ a curve parametrized by arc length, that is $\left|\gamma^{\prime}\right|=1$. Then $\gamma$ is called a Frenet curve of osculating order $r, 1 \leq r \leq m$, if there exists orthonormal vector fields $E_{1}, E_{2}, \ldots, E_{r}$ along $\gamma$ such that $E_{1}=\gamma^{\prime}=T$ and

$$
\nabla_{T} E_{1}=\kappa_{1} E_{2}, \quad \nabla_{T} E_{2}=-\kappa_{1} E_{1}+\kappa_{2} E_{3}, \ldots, \nabla_{T} E_{r}=-\kappa_{r-1} E_{r-1},
$$


where $\kappa_{1}, \ldots, \kappa_{r-1}$ are positive functions on $I$.

A Frenet curve of osculating order 1 is a geodesic; a Frenet curve of osculating order 2 with $\kappa_{1}=$ constant is called a circle; a Frenet curve of osculating order $r, r \geq 3$, with $\kappa_{1}, \ldots, \kappa_{r-1}$ constants is called a helix of order $r$ and helix of order 3 is called, simply, helix.

Let $\left(N^{2 n+1}, \varphi, \xi, \eta, g\right)$ be a Sasakian space form with constant $\varphi$-sectional curvature $c$ and $\gamma: I \rightarrow N$ a non-Legendre Frenet curve of osculating order $r$ with $\eta(T)=f$, where $f$ is a function defined along $\gamma$ and $f \neq 0$. Since

$$
\begin{aligned}
\nabla_{T}^{3} T= & \left(-3 \kappa_{1} \kappa_{1}^{\prime}\right) E_{1}+\left(\kappa_{1}^{\prime \prime}-\kappa_{1}^{3}-\kappa_{1} \kappa_{2}^{2}\right) E_{2}+\left(2 \kappa_{1}^{\prime} \kappa_{2}+\kappa_{1} \kappa_{2}^{\prime}\right) E_{3} \\
& +\kappa_{1} \kappa_{2} \kappa_{3} E_{4}
\end{aligned}
$$

and

$$
\begin{aligned}
R\left(T, \nabla_{T} T\right) T= & \left(-\frac{(c+3) \kappa_{1}}{4}+\frac{(c-1) \kappa_{1}}{4} f^{2}\right) E_{2}-\frac{(c-1)}{4} f f^{\prime} T+\frac{(c-1)}{4} f^{\prime} \xi \\
& -\frac{3(c-1) \kappa_{1}}{4} g\left(E_{2}, \varphi T\right) \varphi T,
\end{aligned}
$$

we get

$$
\begin{aligned}
\tau_{2}(\gamma)= & \nabla_{T}^{3} T-R\left(T, \nabla_{T} T\right) T \\
= & \left(-3 \kappa_{1} \kappa_{1}^{\prime}+\frac{(c-1)}{4} f f^{\prime}\right) E_{1}+\left(\kappa_{1}^{\prime \prime}-\kappa_{1}^{3}-\kappa_{1} \kappa_{2}^{2}+\frac{(c+3) \kappa_{1}}{4}-\frac{(c-1) \kappa_{1}}{4} f^{2}\right) E_{2} \\
& +\left(2 \kappa_{1}^{\prime} \kappa_{2}+\kappa_{1} \kappa_{2}^{\prime}\right) E_{3}+\kappa_{1} \kappa_{2} \kappa_{3} E_{4}-\frac{(c-1)}{4} f^{\prime} \xi+\frac{3(c-1) \kappa_{1}}{4} g\left(E_{2}, \varphi T\right) \varphi T .
\end{aligned}
$$

If $c=1$ then $\gamma$ is proper-biharmonic if and only if

$$
\left\{\begin{array}{l}
\kappa_{1}=\text { constant }>0, \quad \kappa_{2}=\text { constant } \\
\kappa_{1}^{2}+\kappa_{2}^{2}=1 \\
\kappa_{2} \kappa_{3}=0
\end{array}\right.
$$

and we can state the following Theorem.

Theorem 3.2. If $c=1$ then $\gamma$ is proper-biharmonic if and only if either $\gamma$ is a circle with $\kappa_{1}=1$, or $\gamma$ is a helix with $\kappa_{1}^{2}+\kappa_{2}^{2}=1$.

Now, assume that $c \neq 1$. Then $\gamma$ is proper-bihrmonic if and only if

$$
\left\{\begin{array}{l}
\kappa_{1}=\text { constant }>0 \\
\kappa_{1}^{2}+\kappa_{2}^{2}=\frac{c+3}{4}-\frac{c-1}{4} f^{2}-\frac{1}{\kappa_{1}^{2}} \frac{c-1}{4}\left(f^{\prime}\right)^{2}+\frac{3(c-1)}{4}\left(g\left(E_{2}, \varphi T\right)\right)^{2} \\
\kappa_{2}^{\prime}-\frac{1}{\kappa_{1}} \frac{c-1}{4} f^{\prime} \eta\left(E_{3}\right)+\frac{3(c-1)}{4} g\left(E_{2}, \varphi T\right) g\left(E_{3}, \varphi T\right)=0 \\
\kappa_{2} \kappa_{3}-\frac{1}{\kappa_{1}} \frac{c-1}{4} f^{\prime} \eta\left(E_{4}\right)+\frac{3(c-1)}{4} g\left(E_{2}, \varphi T\right) g\left(E_{4}, \varphi T\right)=0
\end{array}\right.
$$

since, from $\eta(T)=g(T, \xi)=f$ and the first Frenet equation, we get $g\left(\nabla_{T} T, \xi\right)=$ $g\left(\kappa_{1} E_{2}, \xi\right)=f^{\prime}$.

Obviously, the above equations are simpler when $f=\eta(T)=\cos \beta_{1}$ is a constant, where $\beta_{1} \in(0, \pi) \backslash\left\{\frac{\pi}{2}\right\}$ is the angle between the tangent vector field $T$ and the characteristic vector field $\xi$. 
In the following, we will study only this special case. We have

Theorem 3.3. Let $c \neq 1$ and $\gamma$ a Frenet curve of osculating order $r$ such that $\eta(T)=\cos \beta_{1}=$ constant $\notin\{-1,0,1\}$. Then $\gamma$ is proper-biharmonic if and only if either

a) $\gamma$ is a circle with $\varphi T= \pm \sin \beta_{1} E_{2}$ and $\kappa_{1}^{2}=1+(c-1) \sin ^{2} \beta_{1}>0$,

or

b) $\gamma$ is a helix with $\varphi T= \pm \sin \beta_{1} E_{2}$ and $\kappa_{1}^{2}+\kappa_{2}^{2}=1+(c-1) \sin ^{2} \beta_{1}>0$,

or

c) $\gamma$ is a Frenet curve of osculating order $r$, where $r \geq 4$, with

$$
\varphi T=\sin \beta_{1} \cos \beta_{2} E_{2}+\sin \beta_{1} \sin \beta_{2} E_{4}
$$

and

$$
\left\{\begin{array}{l}
\kappa_{1}=\text { constant }>0, \quad \kappa_{2}=\text { constant } \\
\kappa_{1}^{2}+\kappa_{2}^{2}=\frac{c+3}{4}-\frac{c-1}{4} \cos ^{2} \beta_{1}+\frac{3(c-1)}{4} \sin ^{2} \beta_{1} \cos ^{2} \beta_{2} \\
\kappa_{2} \kappa_{3}=-\frac{3(c-1)}{8} \sin ^{2} \beta_{1} \sin \left(2 \beta_{2}\right)
\end{array}\right.
$$

where $\beta_{2} \in(0,2 \pi)$ is a constant such that $c+3-(c-1) \cos ^{2} \beta_{1}+3(c-1) \sin ^{2} \beta_{1} \cos ^{2} \beta_{2}$ $>0,3(c-1) \sin \left(2 \beta_{2}\right)<0$ and $n \geq 2$.

Proof. First, we see that $\eta\left(E_{2}\right)=g\left(E_{2}, \xi\right)=\frac{1}{\kappa_{1}} f^{\prime}=0$. Next, assume that $g\left(E_{2}, \varphi T\right)$ $=\alpha$, where $\alpha$ is a function defined along $\gamma$. Then, using the second Frenet equation, one obtains

$$
\alpha^{\prime}=g\left(\nabla_{T} E_{2}, \varphi T\right)+g\left(E_{2}, \nabla_{T} \varphi T\right)=\kappa_{2} g\left(E_{3}, \varphi T\right)+g\left(E_{2}, \kappa_{1} \varphi E_{2}+\xi-f T\right),
$$

and, since the second term in the right side vanishes, it follows $\kappa_{2} g\left(E_{3}, \varphi T\right)=\alpha^{\prime}$. Now, if $\gamma$ is proper-biharmonic, replacing into the third equation of (3.2) we obtain

$$
\kappa_{2} \kappa_{2}^{\prime}+\frac{3(c-1)}{4} \alpha \alpha^{\prime}=0
$$

and then

$$
\kappa_{2}^{2}+\frac{3(c-1)}{4} \alpha^{2}+\omega_{0}=0
$$

where $\omega_{0}$ is a constant. The second equation of (3.2) becomes

$$
\kappa_{1}^{2}+\kappa_{2}^{2}=\frac{c+3}{4}-\frac{c-1}{4} f^{2}-\kappa_{2}^{2}-\omega_{0} .
$$

Hence $\kappa_{2}=$ constant and $\alpha=$ constant. If $\kappa_{2}=0$ then, from the biharmonic equation $\tau_{2}(\gamma)=0$, we get $E_{2} \| \varphi T$ and, since $g(\varphi T, \varphi T)=1-f^{2}=\sin ^{2} \beta_{1}$ it follows $\varphi T= \pm \sin \beta_{1} E_{2}$. Hence $\gamma$ is a circle with

$$
\kappa_{1}^{2}=\frac{c+3}{4}-\frac{c-1}{4} \cos ^{2} \beta_{1}+\frac{3(c-1)}{4} \sin ^{2} \beta_{1}=1+(c-1) \sin ^{2} \beta_{1} .
$$

Assume that $\kappa_{2} \neq 0$. Then $g\left(E_{3}, \varphi T\right)=0$ and, if $\kappa_{3}=0$ then $\gamma$ is a helix with

$$
\kappa_{1}^{2}+\kappa_{2}^{2}=\frac{c+3}{4}-\frac{c-1}{4} \cos ^{2} \beta_{1}+\frac{3(c-1)}{4} \sin ^{2} \beta_{1}=1+(c-1) \sin ^{2} \beta_{1},
$$

since, using again the biharmonic equation, one obtains $E_{2} \| \varphi T$ in this case too. Next, let $\gamma$ be a proper-biharmonic Frenet curve of osculating order $r$ with $r \geq 4$. Then $g\left(E_{3}, \varphi T\right)=0$ and, from the biharmonic equation we have $\varphi T \in \operatorname{span}\left\{E_{2}, E_{4}\right\}$. Since

$$
g(\varphi T, \varphi T)=1-f^{2}=\sin ^{2} \beta_{1}
$$


it follows

$$
\varphi T=\sin \beta_{1} \cos \beta_{2} E_{2}+\sin \beta_{1} \sin \beta_{2} E_{4},
$$

where

$$
g\left(E_{2}, \varphi T\right)=\alpha=\sin \beta_{1} \cos \beta_{2} \quad \text { and } \quad g\left(E_{4}, \varphi T\right)=\sin \beta_{1} \sin \beta_{2}
$$

with $\beta_{2}=$ constant $\in(0,2 \pi)$.

Finally, if the dimension of $N$ is equal to 3 we can consider an orthogonal system of vectors $\{E=T-f \xi, \varphi T, \xi\}$ along $\gamma$ and, since $f$ is a constant, it follows easily that $\nabla_{T} T \| \varphi T$. Hence $E_{2} \| \varphi T$ in this case.

A special role in the biharmonic equation $\tau_{2}(\gamma)=0$ is played by $g\left(E_{2}, \varphi T\right)$. In the following, we consider $\gamma$ to be a Frenet curve of osculating order $r$, with $\eta(T)=f(s)=\cos \beta(s)$ not necessarily constant, such that $E_{2} \perp \varphi T$ or $E_{2} \| \varphi T$.

Case I: $\mathbf{c} \neq \mathbf{1}, \mathbf{E}_{\mathbf{2}} \perp \varphi \mathbf{T}$.

In this case $\gamma$ is proper-biharmonic if and only if

$$
\left\{\begin{array}{l}
\kappa_{1}=\text { constant }>0, \\
\kappa_{1}^{2}+\kappa_{2}^{2}=\frac{c+3}{4}-\frac{c-1}{4} f^{2}-\frac{1}{\kappa_{1}^{2}} \frac{c-1}{4}\left(f^{\prime}\right)^{2} \\
\kappa_{2}^{\prime}-\frac{1}{\kappa_{1}} \frac{c-1}{4} f^{\prime} \eta\left(E_{3}\right)=0 \\
\kappa_{2} \kappa_{3}-\frac{1}{\kappa_{1}} \frac{c-1}{4} f^{\prime} \eta\left(E_{4}\right)=0
\end{array}\right.
$$

From $g\left(E_{2}, \xi\right)=\frac{1}{\kappa_{1}} f^{\prime}$ one obtains $g\left(\nabla_{T} E_{2}, \xi\right)-g\left(E_{2}, \varphi T\right)=\frac{1}{\kappa_{1}} f^{\prime \prime}$ and then $\kappa_{2} \eta\left(E_{3}\right)$ $=\frac{1}{\kappa_{1}} f^{\prime \prime}+\kappa_{1} f$. Replacing into the third equation of (3.3) one obtains

$$
\kappa_{2} \kappa_{2}^{\prime}-\frac{1}{\kappa_{1}^{2}} \frac{c-1}{4}\left(f^{\prime} f^{\prime \prime}+\kappa_{1}^{2} f f^{\prime}\right)=0
$$

and then

$$
\kappa_{2}^{2}-\frac{1}{\kappa_{1}^{2}} \frac{c-1}{4}\left(\left(f^{\prime}\right)^{2}+\kappa_{1}^{2} f^{2}\right)+\omega_{1}=0,
$$

where $\omega_{1}$ is a constant. Now, from the second equation of (3.3) we have

$$
\kappa_{1}^{2}+\kappa_{2}^{2}=\frac{c+3}{4}-\kappa_{2}^{2}-\omega_{1} .
$$

Hence $\kappa_{2}=$ constant and $\left(f^{\prime \prime}+\kappa_{1}^{2} f\right) f^{\prime}=0$.

Now, using the Frenet equations, from $g\left(E_{2}, \varphi T\right)=0$ one obtains $\kappa_{2} g\left(E_{3}, \varphi T\right)=$ $-\frac{1}{\kappa_{1}} f^{\prime}$ and then, from $\kappa_{2} g\left(E_{3}, \xi\right)=\frac{1}{\kappa_{1}} f^{\prime \prime}+\kappa_{1} f$, we get

$$
\kappa_{2} \kappa_{3} g\left(E_{4}, \xi\right)=\frac{1}{\kappa_{1}}\left(f^{\prime \prime \prime}+\left(\kappa_{1}^{2}+\kappa_{2}^{2}\right) f^{\prime}\right) .
$$

Since $\tau_{2}(\gamma)=0$ implies $\eta\left(\tau_{2}(\gamma)\right)=0$ one obtains, after a straightforward computation that $f^{\prime} f^{\prime \prime \prime}=0$. Using this result and differentiating $\left(f^{\prime \prime}+\kappa_{1}^{2} f\right) f^{\prime}=0$ along $\gamma$ we have

$$
\kappa_{1}^{2}\left(f^{\prime}\right)^{2}+\left(f^{\prime \prime}+\kappa_{1}^{2} f\right) f^{\prime \prime}=0 .
$$

We just obtained that $f=$ constant.

We can state 
Theorem 3.4. Assume that $c \neq 1, n \geq 2$ and $\nabla_{T} T \perp \varphi T$. Then $\gamma$ is properbiharmonic if and only if either

a) $\gamma$ is a circle with $\eta(T)=\cos \beta_{0}$ and $\kappa_{1}^{2}=\frac{c+3}{4}-\frac{c-1}{4} \cos ^{2} \beta_{0}$,

or

b) $\gamma$ is a helix with $\eta(T)=\cos \beta_{0}$ and $\kappa_{1}^{2}+\kappa_{2}^{2}=\frac{c+3}{4}-\frac{c-1}{4} \cos ^{2} \beta_{0}$, where $\beta_{0} \in(0,2 \pi) \backslash\left\{\frac{\pi}{2}, \pi, \frac{3 \pi}{2}\right\}$ is a constant such that $\frac{c+3}{4}-\frac{c-1}{4} \cos ^{2} \beta_{0}>0$.

Remark 3.5. We note that the biharmonic equation $\tau_{2}(\gamma)=0$ for curves $\gamma$ with $\nabla_{T} T \perp \varphi T$ is equivalent to

$$
\Delta H=\frac{1}{4}\left(c+3-(c-1) \cos ^{2} \beta_{0}\right) H,
$$

i.e. $H$ is an eigenvector of $\Delta$, where $H=\nabla_{T} T$ is the mean curvature vector field of $\gamma$.

Case II: $\mathbf{c} \neq \mathbf{1}, \mathbf{E}_{\mathbf{2}} \| \varphi \mathbf{T}$.

In this case $g\left(E_{2}, \xi\right)=\frac{1}{\kappa_{1}} f^{\prime}=0$ and then $f=\cos \beta_{0}=$ constant. Since $g(\varphi T, \varphi T)=$ $1-(g(T, T))^{2}=\sin ^{2} \beta_{0}$ we have $\varphi T= \pm \sin \beta_{0} E_{2}$.

We obtain

Proposition 3.6. Assume that $c \neq 1$ and $\nabla_{T} T \| \varphi T$. Then $\gamma$ is proper-biharmonic if and only if either

a) $\gamma$ is a circle with $\eta(T)=\cos \beta_{0}$ and $\kappa_{1}^{2}=c-(c-1) \cos ^{2} \beta_{0}$,

or

b) $\gamma$ is a helix with $\eta(T)=\cos \beta_{0}$ and $\kappa_{1}^{2}+\kappa_{2}^{2}=c-(c-1) \cos ^{2} \beta_{0}$, where $\beta_{0} \in(0,2 \pi) \backslash\left\{\frac{\pi}{2}, \pi, \frac{3 \pi}{2}\right\}$ is a constant such that $c-(c-1) \cos ^{2} \beta_{0}>0$.

Next, let $\gamma$ be a proper-biharmonic non-Legendre curve with $\nabla_{T} T \| \varphi T$. As $\varphi T=$ $\pm \sin \beta_{0} E_{2}$ one obtains after a straightforward computation that

$$
\nabla_{T} E_{2}=-\frac{1}{\sin \beta_{0}}\left(\frac{\kappa_{1}}{\sin \beta_{0}} \pm \cos \beta_{0}\right) T+\frac{1}{\sin \beta_{0}}\left(\frac{\kappa_{1} \cos \beta_{0}}{\sin \beta_{0}} \pm 1\right) \xi
$$

Using the second Frenet equation we have

$$
\kappa_{2}^{2}=\frac{\left(\kappa_{1} \cos \beta_{0} \pm \sin \beta_{0}\right)^{2}}{\sin ^{2} \beta_{0}} .
$$

Thus $\gamma$ is a circle if and only if $\kappa_{1}=\mp \tan \beta_{0}>0$. From Proposition 3.6 we easily get that $\gamma$ is a proper-biharmonic circle if and only if

$$
\kappa_{1}^{2}=\frac{c-1+\sqrt{c^{2}-2 c+5}}{2} \text { and } \cos ^{2} \beta_{0}=\frac{c+1-\sqrt{c^{2}-2 c+5}}{2(c-1)} .
$$

If $\kappa_{2} \neq 0$, from the expression of $\kappa_{2}$ and the third Frenet equation it follows that $\kappa_{3}=0$. Hence $\gamma$ is a helix. Now, $\gamma$ is proper-biharmonic if and only if $\kappa_{1}$ satisfies

$$
\kappa_{1}^{2} \pm \cos \left(2 \beta_{0}\right) \kappa_{1}+(1-c) \sin ^{4} \beta_{0}=0
$$

and $\beta_{0} \in(0,2 \pi) \backslash\left\{\frac{\pi}{2}, \pi, \frac{3 \pi}{2}\right\}$ if $c>1$ or $\beta_{0} \in(0,2 \pi) \backslash\left\{\frac{\pi}{2}, \pi, \frac{3 \pi}{2}\right\}$ such that $\cos \beta_{0} \in$ $\left(-\sqrt{\frac{c-1}{c-2}}, \sqrt{\frac{c-1}{c-2}}\right)$ if $c<1$.

We conclude with the following

Theorem 3.7. If $c \neq 1$ and $\nabla_{T} T \| \varphi T$, then $\gamma$ is a Frenet curve of osculating order $r \leq 3$ and it is proper-biharmonic if and only if either

a) $\gamma$ is a circle with $\eta(T)= \pm \sqrt{\frac{c+1-\sqrt{c^{2}-2 c+5}}{2(c-1)}}$ and $\kappa_{1}^{2}=\frac{c-1+\sqrt{c^{2}-2 c+5}}{2}$, or 
b) $\gamma$ is a helix with $\eta(T)=\cos \beta_{0}$ and $\kappa_{1}$ satisfies

$$
\kappa_{1}^{2} \pm \cos \left(2 \beta_{0}\right) \kappa_{1}+(1-c) \sin ^{4} \beta_{0}=0,
$$

where $\beta_{0}=$ constant $\in(0,2 \pi) \backslash\left\{\frac{\pi}{2}, \pi, \frac{3 \pi}{2}\right\}$ if $c>1$ or $\beta_{0}=$ constant $\in(0,2 \pi) \backslash$ $\left\{\frac{\pi}{2}, \pi, \frac{3 \pi}{2}\right\}$ such that $\cos \beta_{0} \in\left(-\sqrt{\frac{c-1}{c-2}}, \sqrt{\frac{c-1}{c-2}}\right)$ if $c<1$. In the last case $\kappa_{2}^{2}=$ $\left(\kappa_{1} \cot \beta_{0} \pm 1\right)^{2}$.

Remark 3.8. A curve $\gamma$ with $\nabla_{T} T \| \varphi T$ is proper-biharmonic if and only if

$$
\Delta H=\left(c-(c-1) \cos ^{2} \beta_{0}\right) H,
$$

where $H$ is the mean curvature vector field of $\gamma$.

\section{Biharmonic CURves in $\mathbb{R}^{2 n+1}(-3)$}

While proper-biharmonic Legendre curves exist only in a Sasakian space form $N^{2 n+1}(c)$ with constant $\varphi$-sectional curvature $c$ bigger than 1 if $n=1$, or -3 if $n>1$ (see [12], 10]), proper-biharmonic non-Legendre curves can be found in Sasakian space forms with any $\varphi$-sectional curvature.

We mention that, in the case when $c=-3$, T. Sasahara studied in [16] the submanifolds in the Sasakian space form $\mathbb{R}^{2 n+1}(-3)$ whose $\varphi$-mean curvature vectors are eigenvectors of the Laplacian and in [17] the Legendre surfaces in $\mathbb{R}^{5}(-3)$ for which mean curvature vectors field are eigenvectors of the Laplacian.

In this section we obtain the explicit equations for proper-biharmonic circles with $E_{2} \perp \varphi T$ and for all proper-biharmonic curves with $E_{2} \| \varphi T$ in $\mathbb{R}^{2 n+1}(-3)$.

First, let us recall briefly some notions and results about the structure of the Sasakian space form $\mathbb{R}^{2 n+1}(-3)$ as they are presented in $[3$.

Consider on $\mathbb{R}^{2 n+1}(-3)$, with elements of the form $\left(x^{1}, \ldots, x^{n}, y^{1}, \ldots, y^{n}, z\right)$, its standard contact structure defined by the 1-form $\eta=\frac{1}{2}\left(d z-\sum_{i=1}^{n} y^{i} d x^{i}\right)$, the characteristic vector field $\xi=2 \frac{\partial}{\partial z}$ and the tensor field $\varphi$ given by the matrix

$$
\left(\begin{array}{ccc}
0 & \delta_{i j} & 0 \\
-\delta_{i j} & 0 & 0 \\
0 & y^{j} & 0
\end{array}\right)
$$

Then $g=\eta \otimes \eta+\frac{1}{4} \sum_{i=1}^{n}\left(\left(d x^{i}\right)^{2}+\left(d y^{i}\right)^{2}\right)$ is an associated Riemannian metric and $\left(\mathbb{R}^{2 n+1}, \varphi, \xi, \eta, g\right)$ is a Sasakian space form with constant $\varphi$-sectional curvature equal to -3 , denoted $\mathbb{R}^{2 n+1}(-3)$.

The vector fields $X_{i}=2 \frac{\partial}{\partial y^{i}}, X_{n+i}=\varphi X_{i}=2\left(\frac{\partial}{\partial x^{i}}+y^{i} \frac{\partial}{\partial z}\right), i=1, \ldots, n$, and $\xi=2 \frac{\partial}{\partial z}$ form an orthonormal basis in $\mathbb{R}^{2 n+1}(-3)$ and after straightforward computations one obtains

$$
\left[X_{i}, X_{j}\right]=\left[X_{n+i}, X_{n+j}\right]=\left[X_{i}, \xi\right]=\left[X_{n+i}, \xi\right]=0, \quad\left[X_{i}, X_{n+j}\right]=2 \delta_{i j} \xi
$$

and

$$
\begin{gathered}
\nabla_{X_{i}} X_{j}=\nabla_{X_{n+i}} X_{n+j}=0, \quad \nabla_{X_{i}} X_{n+j}=\delta_{i j} \xi, \quad \nabla_{X_{n+i}} X_{j}=-\delta_{i j} \xi, \\
\nabla_{X_{i}} \xi=\nabla_{\xi} X_{i}=-X_{n+i}, \quad \nabla_{X_{n+i}} \xi=\nabla_{\xi} X_{n+i}=X_{i}
\end{gathered}
$$

for any $i, j=1, \ldots, n$.

Now, let $\gamma: I \rightarrow \mathbb{R}^{2 n+1}(-3)$ be a Frenet curve of osculating order $r>1$, parametrized by arc length, with the tangent vector field $T=\gamma^{\prime}$ given by

$$
T=\sum_{i=1}^{n}\left(T_{i} X_{i}+T_{n+i} X_{n+i}\right)+\cos \beta_{0} \xi
$$


where $\cos \beta_{0}$ is a constant. Using the above formulas for the Levi-Civita connection we have

$$
\nabla_{T} T=\sum_{i=1}^{n}\left(\left(T_{i}^{\prime}+2 \cos \beta_{0} T_{n+i}\right) X_{i}+\left(T_{n+i}^{\prime}-2 \cos \beta_{0} T_{i}\right) X_{n+i}\right)
$$

From Theorems 3.4 and 3.7, using the same techniques as in [4, [5] and [7], we get

Theorem 4.1. The parametric equations of proper-biharmonic circles parametrized by arc length in $\mathbb{R}^{2 n+1}(-3), n \geq 2$, with $\nabla_{T} T \perp \varphi T$, are

$$
\left\{\begin{aligned}
x^{i}(s)= & \pm \frac{1}{\kappa_{1}}\left(2 \sin \left(\kappa_{1} s\right) c_{1}^{i} \mp 2 \cos \left(\kappa_{1} s\right) c_{2}^{i}-\cos \left(2 \kappa_{1} s\right) d_{1}^{i}\right. \\
& \left.-\sin \left(2 \kappa_{1} s\right) d_{2}^{i}\right)+a^{i} \\
y^{i}(s)= & \frac{1}{\kappa_{1}}\left(2 \cos \left(\kappa_{1} s\right) c_{1}^{i} \pm 2 \sin \left(\kappa_{1} s\right) c_{2}^{i}+\sin \left(2 \kappa_{1} s\right) d_{1}^{i}\right. \\
& \left.-\cos \left(2 \kappa_{1} s\right) d_{2}^{i}\right)+b^{i} \\
z(s)= & \pm \frac{2}{\kappa_{1}}\left(1+\sum_{i=1}^{n}\left(\left(c_{1}^{i}\right)^{2}+\left(c_{2}^{i}\right)^{2}\right)\right) s \\
& +\frac{1}{2 \kappa_{1}^{2}} \sum_{i=1}^{n}\left( \pm \cos \left(4 \kappa_{1} s\right) d_{1}^{i} d_{2}^{i}-2 \cos \left(2 \kappa_{1} s\right) c_{1}^{i} c_{2}^{i}\right. \\
& \left.+4 \cos \left(3 \kappa_{1} s\right) c_{2}^{i} d_{2}^{i}-4 \sin \left(3 \kappa_{1} s\right) c_{1}^{i} d_{2}^{i}\right) \\
& \mp \frac{1}{\kappa_{1}} \sum_{i=1}^{n} b^{i}\left(-2 \sin \left(\kappa_{1} s\right) c_{1}^{i} \pm 2 \cos \left(\kappa_{1} s\right) c_{2}^{i}\right. \\
& \left.+\cos \left(2 \kappa_{1} s\right) d_{1}^{i}+\sin \left(2 \kappa_{1} s\right) d_{2}^{i}\right)+e
\end{aligned}\right.
$$

where $\kappa_{1}^{2}=\cos ^{2} \beta_{0}, \beta_{0} \in(0,2 \pi) \backslash\left\{\frac{\pi}{2}, \pi, \frac{3 \pi}{2}\right\}$ is a constant, and $a^{i}, b^{i}, c_{1}^{i}, c_{2}^{i}, d_{1}^{i}$, $d_{2}^{i}$ and $e$ are constants such that the $n$-dimensional constant vectors $c_{j}=\left(c_{j}^{1}, \ldots, c_{j}^{n}\right)$ and $d_{j}=\left(d_{j}^{1}, \ldots, d_{j}^{n}\right), j=1,2$, satisfy

$$
\left\{\begin{array}{l}
\left|c_{1}\right|^{2}+\left|c_{2}\right|^{2}+\left|d_{1}\right|^{2}+\left|d_{2}\right|^{2}=\sin ^{2} \beta_{0} \\
\left\langle c_{1}, d_{1}\right\rangle \pm\left\langle c_{2}, d_{2}\right\rangle=0,\left\langle c_{1}, d_{2}\right\rangle \mp\left\langle c_{2}, d_{1}\right\rangle=0
\end{array} .\right.
$$

Proof. Let $\gamma: I \rightarrow \mathbb{R}^{2 n+1}(-3)$ be a circle parametrized by arc length, with the tangent vector field $T=\gamma^{\prime}$ given by (4.1) and $\nabla_{T} T \perp \varphi T$. From the equation (4.2) one obtains

$$
E_{2}=\frac{1}{\kappa_{1}} \sum_{i=1}^{n}\left(\left(T_{i}^{\prime}+2 \cos \beta_{0} T_{n+i}\right) X_{i}+\left(T_{n+i}^{\prime}-2 \cos \beta_{0} T_{i}\right) X_{n+i}\right)
$$

and, using $g\left(E_{2}, \varphi T\right)=0$, a direct computation shows that

$$
\begin{aligned}
\nabla_{T} E_{2}= & \frac{1}{\kappa_{1}}\left(\sum_{i=1}^{n}\left(\left(T_{i}^{\prime}+2 \cos \beta_{0} T_{n+i}\right)^{\prime}+\left(T_{n+i}^{\prime}-2 \cos \beta_{0} T_{i}\right) \cos \beta_{0}\right) X_{i}\right. \\
& \left.+\left(\left(T_{n+i}^{\prime}-2 \cos \beta_{0} T_{i}\right)^{\prime}-\left(T_{i}^{\prime}+2 \cos \beta_{0} T_{n+i}\right) \cos \beta_{0}\right) X_{n+i}\right)
\end{aligned}
$$

and, since $\gamma$ is a circle, it follows

$$
\left\{\begin{array}{c}
A_{i}^{\prime}+B_{i} \cos \beta_{0}=0 \\
B_{i}^{\prime}-A_{i} \cos \beta_{0}=0
\end{array},\right.
$$


where $A_{i}=\frac{1}{\kappa_{1}}\left(T_{i}^{\prime}+2 \cos \beta_{0} T_{n+i}\right)$ and $B_{i}=\frac{1}{\kappa_{1}}\left(T_{n+i}^{\prime}-2 \cos \beta_{0} T_{i}\right)$.

Solving (4.4) and imposing for $\gamma$ to be proper-biharmonic, according to Theorem 3.4 that is $\kappa_{1}= \pm \cos \beta_{0}>0$, we get the following equations

$$
\left\{\begin{array}{c}
T_{i}^{\prime} \pm 2 \kappa_{1} T_{n+i}=\kappa_{1} \cos \left(\kappa_{1} s\right) c_{1}^{i} \pm \kappa_{1} \sin \left(\kappa_{1} s\right) c_{2}^{i} \\
T_{n+i}^{\prime} \mp 2 \kappa_{1} T_{i}= \pm \kappa_{1} \sin \left(\kappa_{1} s\right) c_{1}^{i}-\kappa_{1} \cos \left(\kappa_{1} s\right) c_{2}^{i}
\end{array}\right.
$$

which general solutions are

$$
\left\{\begin{array}{c}
T_{i}=-\sin \left(\kappa_{1} s\right) c_{1}^{i} \pm \cos \left(\kappa_{1} s\right) c_{2}^{i}+\cos \left(2 \kappa_{1} s\right) d_{1}^{i}+\sin \left(2 \kappa_{1} s\right) d_{2}^{i} \\
T_{n+i}= \pm \cos \left(\kappa_{1} s\right) c_{1}^{i}+\sin \left(\kappa_{1} s\right) c_{2}^{i} \pm \sin \left(2 \kappa_{1} s\right) d_{1}^{i} \mp \cos \left(2 \kappa_{1} s\right) d_{2}^{i}
\end{array}\right.
$$

where $c_{1}^{i}, c_{2}^{i}, d_{1}^{i}$ and $d_{2}^{i}$ are constants, such that

$$
\left\{\begin{array}{l}
\sum_{i=1}^{n}\left(\left(c_{1}^{i}\right)^{2}+\left(c_{2}^{i}\right)^{2}+\left(d_{1}^{i}\right)^{2}+\left(d_{2}^{i}\right)^{2}\right)=\sin ^{2} \beta_{0} \\
\sum_{i=1}^{n}\left(\left(c_{1}^{i}\right)\left(d_{1}^{i}\right) \pm\left(c_{2}^{i}\right)\left(d_{2}^{i}\right)\right)=0, \quad \sum_{i=1}^{n}\left(\left(c_{1}^{i}\right)\left(d_{2}^{i}\right) \mp\left(c_{2}^{i}\right)\left(d_{1}^{i}\right)\right)=0
\end{array},\right.
$$

since $g(T, T)=1$.

Finally, replacing into expression of $\gamma^{\prime}$ and integrating we get (4.3).

Remark 4.2. In order to find explicit examples of proper-biharmonic curves with $\nabla_{T} T \perp \varphi T$ in $\mathbb{R}^{2 n+1}(-3)$ we will stick at proper-biharmonic circles since the computations in the case of helices are rather complicated.

Theorem 4.3. Proper-biharmonic curves in $\mathbb{R}^{2 n+1}(-3)$, with $\nabla_{T} T \| \varphi T$, are either a) Proper-biharmonic circles given by

$$
\left\{\begin{aligned}
x^{i}(s)= & (\sqrt{5}+1)\left(\cos \left(\frac{\sqrt{5}-1}{2} s\right) c_{1}^{i}+\sin \left(\frac{\sqrt{5}-1}{2} s\right) c_{2}^{i}\right)+a^{i} \\
y^{i}(s)= & (\sqrt{5}+1)\left(\sin \left(\frac{\sqrt{5}-1}{2} s\right) c_{1}^{i}-\cos \left(\frac{\sqrt{5}-1}{2} s\right) c_{2}^{i}\right)+b^{i} \\
z(s)= & \frac{1-\sqrt{5} \pm 2 \sqrt{1+\sqrt{5}}}{2} s+\frac{3+\sqrt{5}}{2} \sum_{i=1}^{n}\left(\left(\left(c_{1}^{i}\right)^{2}-\left(c_{2}^{i}\right)^{2}\right) \sin ((\sqrt{5}-1) s)\right. \\
& \left.-2 \cos ((\sqrt{5}-1) s) c_{1}^{i} c_{2}^{i}\right)+(1+\sqrt{5}) \sum_{i=1}^{n} b_{i}\left(\sin \left(\frac{\sqrt{5}-1}{2} s\right) c_{2}^{i}\right. \\
& \left.+\cos \left(\frac{\sqrt{5}-1}{2} s\right) c_{1}^{i}\right)+d
\end{aligned}\right.
$$

where $a^{i}, b^{i}, c_{1}^{i}, c_{2}^{i}$ and $d$ are constants such that the $n$-dimensional constant vectors $c_{j}=\left(c_{j}^{1}, \ldots, c_{j}^{n}\right), j=1,2$, satisfy

$$
\left|c_{1}\right|^{2}+\left|c_{2}\right|^{2}=\frac{3-\sqrt{5}}{4}
$$


b) Proper-biharmonic helices given by

$$
\left\{\begin{aligned}
x^{i}(s)= & -\frac{2 \kappa_{1}}{\kappa_{1} \pm \sin \left(2 \beta_{0}\right)}\left(\cos \left(\frac{\kappa_{1} \pm \sin \left(2 \beta_{0}\right)}{\kappa_{1}} s\right) c_{1}^{i}+\sin \left(\frac{\kappa_{1} \pm \sin \left(2 \beta_{0}\right)}{\kappa_{1}} s\right) c_{2}^{i}\right)+a^{i} \\
y^{i}(s)= & \frac{2 \kappa_{1}}{\kappa_{1} \pm \sin \left(2 \beta_{0}\right)}\left(\sin \left(\frac{\kappa_{1} \pm \sin \left(2 \beta_{0}\right)}{\kappa_{1}} s\right) c_{1}^{i}-\cos \left(\frac{\kappa_{1} \pm \sin \left(2 \beta_{0}\right)}{\kappa_{1}} s\right) c_{2}^{i}\right)+b^{i} \\
z(s)= & 2\left(\cos \beta_{0}+\frac{\kappa_{1} \sin ^{2} \beta_{0}}{\kappa_{1} \pm \sin \left(2 \beta_{0}\right)}\right) s+\frac{\kappa_{1}^{2}}{\left(\kappa_{1} \pm \sin \left(2 \beta_{0}\right)\right)^{2}} \\
& \cdot\left(\sin \left(\frac{2\left(\kappa_{1} \pm \sin \left(2 \beta_{0}\right)\right)}{\kappa_{1}} s\right) \sum_{i=1}^{n}\left(\left(c_{1}^{i}\right)^{2}-\left(c_{2}^{i}\right)^{2}\right)\right. \\
& \left.+\cos \left(\frac{2\left(\kappa_{1} \pm \sin \left(2 \beta_{0}\right)\right)}{\kappa_{1}} s\right) \sum_{i=1}^{n}\left(c_{1}^{i} c_{2}^{i}\right)\right) \\
& -\frac{2 \kappa_{1}}{\kappa_{1} \pm \sin \left(2 \beta_{0}\right)} \sum_{i=1}^{n} b^{i}\left(\cos \left(\frac{\kappa_{1} \pm \sin \left(2 \beta_{0}\right)}{\kappa_{1}} s\right) c_{1}^{i}\right. \\
& \left.+\sin \left(\frac{\kappa_{1} \pm \sin \left(2 \beta_{0}\right)}{\kappa_{1}} s\right) c_{2}^{i}\right)+d
\end{aligned}\right.
$$

where $\beta_{0} \in(0,2 \pi) \backslash\left\{\frac{\pi}{2}, \pi, \frac{3 \pi}{2}\right\}$ is a constant such that $\cos \beta_{0} \in\left(-1,-\frac{2 \sqrt{5}}{5}\right) \cup\left(\frac{2 \sqrt{5}}{5}, 1\right)$, $\kappa_{1}$ is a positive solution of the equation

$$
\kappa_{1}^{2} \pm \sin \left(2 \beta_{0}\right) \kappa_{1}+4 \sin ^{4} \beta_{0}=0
$$

and $a^{i}, b^{i}, c_{1}^{i}, c_{2}^{i}$ and $d$ are constants such that

$$
\left|c_{1}\right|^{2}+\left|c_{2}\right|^{2}=\sin ^{2} \beta_{0} .
$$

Proof. We will prove only the first statement because the second one can be obtained in a similar way by the meaning of Theorem 3.7 .

Assume that $\gamma$ is a proper-biharmonic circle in $\mathbb{R}^{2 n+1}(-3)$ parametrized by arc length, such that $\nabla_{T} T \| \varphi T$. Then, from (4.2) and since $\varphi T=\sum_{i=1}^{n}\left(-T_{n+i} X_{i}+\right.$ $\left.T_{i} X_{n+i}\right), g(\varphi T, \varphi T)=\sin ^{2} \beta_{0}$, where $\eta(T)=\cos \beta_{0}$, one obtains

$$
T_{i}^{\prime}=\left(\mp \frac{\sin \left(2 \beta_{0}\right)}{\kappa_{1}}-1\right) T_{n+i}, \quad T_{n+i}^{\prime}=\left( \pm \frac{\sin \left(2 \beta_{0}\right)}{\kappa_{1}}+1\right) T_{i}
$$

Now, since $\gamma$ is a proper-biharmonic circle we get, from Theorem 3.7, $\kappa_{1}=\mp \tan \beta_{0}>$ 0 and $\cos ^{2} \beta_{0}=\frac{1+\sqrt{5}}{4}$ and hence the above equations become

$$
T_{i}^{\prime}=\frac{\sqrt{5}-1}{2} T_{n+i}, \quad T_{n+i}^{\prime}=\frac{1-\sqrt{5}}{2} T_{i},
$$

with general solutions

$T_{i}=\cos \left(\frac{\sqrt{5}-1}{2}\right) c_{1}^{i}+\sin \left(\frac{\sqrt{5}-1}{2}\right) c_{2}^{i}, \quad T_{n+i}=\cos \left(\frac{\sqrt{5}-1}{2}\right) c_{2}^{i}-\sin \left(\frac{\sqrt{5}-1}{2}\right) c_{1}^{i}$,

where $c_{1}^{i}$ and $c_{2}^{i}, i=1, \ldots, n$, are constants.

Replacing in the expression of $T=\gamma^{\prime}$, integrating and imposing $g(T, T)=1$ we obtain the conclusion. 


\section{REFERENCES}

[1] A. Arvanitoyeorgos, F. Defever, G. Kaimakamis, V.J. Papantoniou. Biharmonic Lorentz hypersurfaces in $E_{1}^{4}$, Pacific J. Math., 229(2007), 293-305.

[2] A. Balmuş, S. Montaldo, C. Oniciuc. Classification results for biharmonic submanifolds in spheres, Israel J. Math., to appear.

[3] D.E. Blair. Riemannian Geometry of Contact and Symplectic Manifolds, Birkhäuser Boston, Progress in Mathematics, Volume 203, 2002.

[4] R. Caddeo, C. Oniciuc, P. Piu. Explicit formulas for non-geodesic biharmonic curves of the Heisenberg group, Rend. Sem. Mat. Univ. e Politec. Torino, 62(2004), 265-278.

[5] R. Caddeo, S. Montaldo, C. Oniciuc, P. Piu. The clasification of biharmonic curves of CartanVranceanu 3-dimensional spaces, Modern Trends in Geometry and Topology, Deva, September 5-11, 2005, 121-131.

[6] B.Y. Chen. A report on submanifolds of finite type, Soochow J. Math., 22(1996), 117-337.

[7] J.T. Cho, J. Inoguchi, J.-E. Lee. Biharmonic curves in 3-dimensional Sasakian space form, Ann. Math. Pura Appl., to appear.

[8] J. Eells, J.H. Sampson. Harmonic mappings of Riemannian manifolds, Amer. J. Math., 86(1964), 109-160.

[9] D. Fetcu, C. Oniciuc. Explicit formulas for biharmonic submanifolds in non-Euclidean 3spheres, Abh. Math. Semin. Univ. Hamburg, 77(2007), 179-190.

[10] D. Fetcu, C. Oniciuc. Explicit formulas for biharmonic submanifolds in Sasakian space forms, Pacific J. Math., 240(1)(2009), 85-107.

[11] T. Ichiyama, J. Inoguchi, H. Urakawa. Bi-harmonic maps and bi-Yang-Mills fields, preprint.

[12] J. Inoguchi. Submanifolds with harmonic mean curvature in contact 3-manifolds, Colloq. Math., 100(2004), 163-179.

[13] G.Y. Jiang. 2-harmonic maps and their first and second variational formulas, Chinese Ann. Math. Ser. A7(4)(1986), 389-402.

[14] S. Montaldo, C. Oniciuc. A short survey on biharmonic maps between Riemannian manifolds, Rev. Un. Mat. Argentina, 47(2)(2006), 1-22.

[15] Y-L. Ou, Z-P. Wang. Biharmonic maps into Sol and Nil spaces, arXiv:math.DG/0612329v1.

[16] T. Sasahara. Submanifolds in a Sasakian manifold $\mathbb{R}^{2 n+1}(-3)$ whose $\phi$-mean curvature vectors are eigenvectors, J. Geom., 75(2002), 166-178.

[17] T. Sasahara. Spectral decomposition of mean curvature vector fields of surfaces in a Sasakian manifold $\mathbb{R}^{2 n+1}(-3)$, Results Math., 43(1-2)(2003), 168-180.

[18] T. Sasahara. Legendre surfaces in Sasakian space forms whose mean curvature vectors are eigenvectors, Publ. Math. Debrecen, 67(3-4)(2005), 285-303.

[19] The Bibliography of Biharmonic Maps. http://people.unica.it/ biharmonic/publication/.

[20] W. Zhang. New examples of biharmonic submanifolds in $\mathbb{C} P^{n}$ and $\mathbb{S}^{2 n+1}$, arXiv:math.DG/0705.3961v1.

Department of Mathematics, "Gh. Asachi" Technical University of Iasi, Bd. Carol I NO. 11, 700506 IAsi, RomaniA

E-mail address: dfetcu@math.tuiasi.ro 